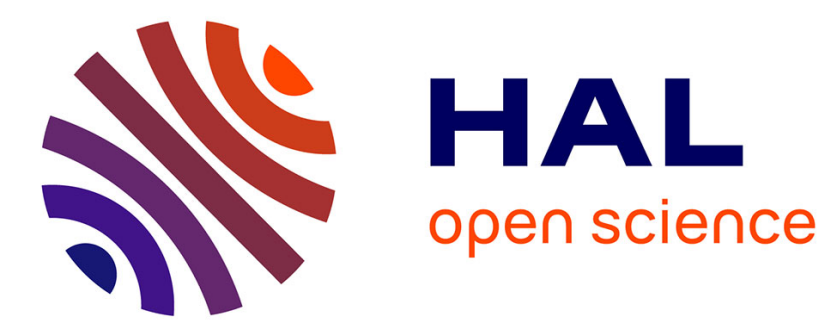

\title{
A branching process model of heterogeneous DNA damages caused by radiotherapy in in vitro cell cultures
}

Thierry Bastogne, Jean-Louis Marchand, Sophie Pinel, Pierre Vallois

\section{To cite this version:}

Thierry Bastogne, Jean-Louis Marchand, Sophie Pinel, Pierre Vallois. A branching process model of heterogeneous DNA damages caused by radiotherapy in in vitro cell cultures. Mathematical Biosciences, 2017, 294, pp.100-109. 10.1016/j.mbs.2017.09.006 . hal-01669004

\section{HAL Id: hal-01669004 https://hal.science/hal-01669004}

Submitted on 8 Jan 2018

HAL is a multi-disciplinary open access archive for the deposit and dissemination of scientific research documents, whether they are published or not. The documents may come from teaching and research institutions in France or abroad, or from public or private research centers.
L'archive ouverte pluridisciplinaire HAL, est destinée au dépôt et à la diffusion de documents scientifiques de niveau recherche, publiés ou non, émanant des établissements d'enseignement et de recherche français ou étrangers, des laboratoires publics ou privés. 


\title{
A Branching Process Model of Heterogeneous DNA Damages caused by Radiotherapy in in vitro Cell Cultures
}

\author{
T. Bastogne ${ }^{c, d, e, *}$, J.-L. Marchand ${ }^{\mathrm{a}, \mathrm{b}, \mathrm{e}}$, S. Pinel ${ }^{\mathrm{c}, \mathrm{d}}$, P. Vallois $\mathrm{s}^{\mathrm{a}, \mathrm{b}, \mathrm{e}}$ \\ ${ }^{a}$ Université de Lorraine, IECN, UMR 7502, Campus Sciences, BP 70239, Vandoeuvre-ls-Nancy Cedex, 54506, France \\ ${ }^{b}$ CNRS, IECN, UMR 7502, France \\ ${ }^{c}$ Université de Lorraine, CRAN, UMR 7039, Campus Sciences, BP 70239, Vandoeuvre-ls-Nancy Cedex, 54506, France \\ ${ }^{d}$ CNRS, CRAN, UMR 7039, France \\ ${ }^{e} I N R I A, B I G S$, France
}

\begin{abstract}
This paper deals with the dynamic modeling and simulation of cell damage heterogeneity and associated mutant cell phenotypes in the therapeutic responses of cancer cell populations submitted to a radiotherapy session during in vitro assays. Each cell is described by a finite number of phenotypic states with possible transitions between them. The population dynamics is then given by an age-dependent multi-type branching process. From this representation, we obtain formulas for the average size of the global survival population as well as the one of subpopulations associated with 10 mutation phenotypes. The proposed model has been implemented into Matlab ${ }^{\circledR}$ and the numerical results corroborate the ability of the model to reproduce four major types of cell responses: delayed growth, anti-proliferative, cytostatic and cytotoxic.
\end{abstract}

Keywords: Branching process, cancer cells, heterogeneity, radiotherapy

\section{Introduction}

Due to the complexity of cancer, integrative biology has taken an important place in oncology research since the beginning of 2000's [2]. Indeed, cancer is the result of inter-dependent multi-scale phenomena. This is why the understanding of its spread is still an unsolved problem. One main question is to better understand the cause and consequences of heterogeneity in cancer [42,33]. Four facets of heterogeneity are generally described: statistical or distributional heterogeneity, epigenetic or environmental heterogeneity, emergence of intrinsic or clonal heterogeneity and the development of clonal subpopulations in a heterogeneous microenvironment $[34,22]$. The main issue addressed here is to describe and simulate the influence of cell damage heterogeneity and associated mutant cell phenotypes in the therapeutic responses of cancer cell populations submitted to a radiotherapy session during in vitro assays. To help biologists and clinicians to answer such a question, mathematical models play a central role through numerical simulations and statistical analyses.

\footnotetext{
* Corresponding author.

Email address: thierry.bastogne@univ-lorraine.fr (T. Bastogne)
} 
To this aim, four main classes of cancer cell models may be considered.

Ballistic models, e.g. the linear-quadratic model, is a first class of mathematical models commonly implemented into clinical treatment planning systems to guide radiotherapeutists to choose the optimum radiation dose to be delivered [50, 14, 21]. Unfortunately, they only compute average doses and do not account for cell heterogeneity.

A second class deals with the kinetics of cancer cell populations. It has a very long history, dating back to the equation of exponential growth, which is based on a small number of differential equations [26, 40]. The Verhulst-Pearl-Reed's logistic curve [38, 39], the Gompertz's function [32, 43], the Bertalanffy's equation [47] and the Fischer's model [18] also belong to this model category. Their main drawback is their lack of biological basis and their implicit assumption that the tumor is an homogeneous set of cancer cells.

A third class of tumor growth models accounts for the biological cell cycle into the mathematical expression. Models proposed by Cox-Woodburry-Myers in [12] and Burns-Tannock in [9] belong to this group. They consider the existence of at least three main cell populations in a tumor: proliferating, quiescent and necrotic cells. Subsequently, the associated representations often rely on compartmental models in which each compartment is associated with each type of cell [46]. For each cell cycle phase, the biological behavior of the cell is described either by differential equations [20,45] or by Mc Kendrick-von Foerster equations taking the age distribution of the cell population into consideration. Unfortunately, this model class does not consider spatial variability in the tumor.

Another class of models aims at accounting interactions between living subpopulations, such as birthdeath processes describing the dynamics of the number of cells of different types, with interactions (for example competitive of Lotka-Volterra interactions in [6]), or diffusion processes describing population densities or biomasses of each populations with interactions in [44].

A fifth model class examines the spatial evolutions of the tumor growth. Bresch et al. in [8] have used diffusion processes and partial derivative equations to describe a viscoelastic mechanical behavior able to account for cellular adhesion. However, a tumor is not a continuous biological medium but rather an aggregate of cells in an extracellular matrix. Subsequently, the multi-agent paradigm seems more suited than partial derivative models $[48,49]$. In this modeling approach, each agent is an autonomous entity associated with each biological cell of the tumor, whose behavior depends both on its current state and its local environment. In $[15,19]$, the agent-based modeling paradigm were used to describe the spatial-temporal organization of tissues in multi-cellular systems such as tumors. In [17], nine in silico axioms were proposed to represent the operating principles realized during characteristic growth of EMT6/Ro mouse mammary tumor spheroids in culture.

A last and important factor of tumor growth modeling deals with the ability to account for cell heterogeneity. In $[4,3,51,52]$, a $3 \mathrm{D}$ multi-scale agent-based model is developed to simulate cancer heterogeneity brain 
tumors. Unfortunately, the associated computational cost is heavy. Several stochastic modeling paradigms have been proposed to describe heterogeneity in tumors such as Markov chains [23, 28, 29, 36], branching processes $[25,35,10,16,37,41,13,31]$ and even stochastic differential equations $[11,7,1]$, but they were all focused on the steady-state responses of cell populations.

In this stochastic modeling context, we investigate the evolutionary dynamics of mutation heterogeneity in the dynamic responses of cell populations. We consider that the survival response of an in vitro cancer cell culture treated by radiotherapy is a superposition of independent dynamics. Each cell is represented by a finite number of phenotypic states with possible transitions between them. The population dynamics is then given by an age-dependent multi-type branching process. From this representation, we formulate the average size of the global survival population as well as the one of subpopulations associated with 10 mutation phenotypes. Our model has been implemented into Matlab ${ }^{\circledR}$ to carry out numerical experiments and to test its ability to reproduce four main types of treatment responses: delayed growth, anti-proliferative, cytostatic and cytotoxic.

This paper is organized as follows. We start by presenting the construction of the model. As mentioned before, we first describe the behaviour of a clone cell. The population model is presented in Section 3, while its implementation and its simulation results are presented and discussed in Section 4.

\section{The cell model}

We suppose that the initial population is composed of clone cells, obtained by replications of one cancer cell with a given phenotype. The latter may change for each cell after individual damages caused by a single radiotherapy session. We assume independence between cells and therefore focus on only one cell. The treatment effect on one cell is decomposed into two phases :

- Direct effects. If a cell is damaged during the radiotherapy session, its characteristics are modified and its new mutation phenotypic state arises during a period of time $\tau_{d}$ after the treatment application.

- Indirect Effects. As a consequence of the direct effects, different mutations states may appear and lead to the cell death more or less shortly.

The model of the cell lineage system is mathematically represented herein by a branching process (see e.g. $[24,5,27,30])$. In such a model it is assumed that, the evolution of a cell only depends on its birth phenotypic state. Its dynamic evolution is represented by two factors: its progeny and its life span. Both follow probabilistic laws, commonly referred to as offspring distribution and life span distribution. In this study, we have chosen an age-dependent multi-type branching process:

- "multi-type", because we consider 10 different possible states or "types" for a living cell; 
- "age-dependent", since the branching time and the new state are correlated.

Let us briefly recall the dynamic of such processes. All the cells behave independently. Let us consider a cell of type $i$ born at time 0 . At the life span $T_{i}$, it gives birth to the offspring $Z^{i}=\left(Z_{1}^{i}, \cdots, Z_{10}^{i}\right)$, where $Z_{j}^{i}$ is the number of new cells of type $j$. The pair $\left(T_{i}, Z^{i}\right)$ is random. The model being age-dependent, then the life span and the progeny can be dependent. However in our model $T_{i}$ belongs to the finite set of integers $\left\{1, \tau_{1}, \tau_{2}, \tau_{m}, \tau_{b}\right\}$. One should notice that such a process is not Markovian since life spans do not follow exponential laws. That leads to additional difficulties to study such dynamics. Let us examine the biological interpretation for each phenotypic state.

\subsection{Phenotypic state coding}

The label of a cell is either equal to 2 (undamaged cell) or a triplet $a b c$ of integers, where :

- $a \in\{0,1,2\}$ refers to the cell level of proliferation;

- $b \in\{0,1\}$ expresses the repair capacity;

- $c \in\{0,1\}$ is the genomic instability.

Here, 10 states of living cells are considered : 2, 210,211, 201, 200,111, 110, 101, 100, 011 and 000 for the dead cells. The missing encodings, 010 and 001 are not considered because of lack of biological interpretation. For instance, the coding 001 would mean that the cell cannot proliferate, nor mutate. Its capacity to be repaired cannot be used, so that 001 is a redundancy of the death state 000 . We now explain the real significance of the parameters $a, b, c$.

Proliferation level. In level $a=0$, the cell cycle is blocked while in the other cases the cell cycle length takes two values: $\tau_{1}$ if $a=1$ (extended cell cycle) and $\tau_{2}$ otherwise (normal cell cycle). Initially, for a normal cell, $a=2$.

Repair capacity. The element $b$ is equal to 1 if the cell is able to be repaired and 0 otherwise. The change of this repair capacity is a potential consequence of the therapy. The reparation process allows a damaged cell to potentially recover its proliferation level; it is an innate capacity that can be transmitted to the lineage.

Genomic instability. The element $c$ is equal to 1 if the cell is able to stop its cycle and to mutate, and null otherwise.

\subsection{Therapy effects modeling}

We assume that initial cells can be either in state 210 or 200 . 


\begin{tabular}{l|l}
\hline Initial state & Possible evolutions \\
\hline \hline 210 & $\begin{array}{l}\text { the cell has not been hit or repaired faithfully the lesions } \\
\text { the cell has been hit and repaired unfaithfully the lesions } \\
\text { the cell is blocked } \\
\text { the cell has been destroyed (necrosis) or suffered from too many } \\
\text { lesions (apoptosis) }\end{array}$ \\
$\begin{array}{l}\text { the cell has not been hit or repaired faithfully the lesions } \\
\text { the cell has been hit and repaired unfaithfully the lesions } \\
\text { the cell has been destroyed (necrosis) or suffered from too many } \\
\text { lesions (apoptosis) }\end{array}$
\end{tabular}

Table 1: Direct effects: first possible phenotypic states after treatment

\subsubsection{Direct effects}

After treatment, a cell has five possible states, as illustrated in Table 1:

- state 2: the cell is not affected by the treatment, proliferates and gives birth to two daughter cells of the same type after a period of time $T$. Due to the lack of synchronicity between cells, $T$ is a random variable with an uniform law on $\left[0, \tau_{2}\right)$, where $\tau_{2}$ denotes the usual cell cycle length;

- states 110 or 100: the cell survives but its state is changed after a time $T$ with a loss of proliferation level. It is assumed that $T$ is random and takes its value in $\left[0, \tau_{d}\right)$, where $\tau_{d}$ is given;

- state 011: the cell is still alive but begins a quiescence cycle at time $T$;

- state 000: the cell is killed.

\subsubsection{Indirect effects: disturbed lineage of a damaged cell}

The treated population becomes more heterogeneous, as described in Table 1. The second and indirect effects stand in the lack of stability of the lineage due to random mutations. Only the cells of type $a b 1$ may stop their cycle and mutate. The following rules allow to describe the possible issues for each type.

Rule 1 (Proliferation rule). A cell of type abc can proliferate if and only if $a \neq 0$. In that case, an abc-cell gives rise to two daughter cells of the same phenotype ab1 (symmetric proliferation), at time $\tau_{2}$ if $a=2$ and $\tau_{1}$ if $a=1$. The new cells inherit the same values for $a$ and $b$ but they are assumed to be unstable independently of the mother's c-state.

Rule 2 (Stable cells behavior). A stable cell ab0 always ends its cycle of period $\tau_{a}$ and then gives birth to two cells of phenotype ab1. The evolutions of all the concerned states, 210, 200, 110 and 100, are presented in Table 2. 


\begin{tabular}{l|ll}
\hline Initial state & Possible evolutions & States of daughters \\
\hline \hline 210 & Faithful replication $\tau_{2}$ later & 211 \\
\hline 200 & Faithful replication $\tau_{2}$ later & 201 \\
\hline 100 & Faithful replication $\tau_{1}$ later & Fa1 \\
\hline 110 & Faithful replication $\tau_{1}$ later & 1111 \\
\hline
\end{tabular}

Table 2: Indirect effects: replication of $a b 0$ phenotypes with $a=1,2$ after damage

Rule 3 (Unstable cells behavior). An unstable cell ab1, except in state 011, can either proliferate or mutate or die. In the first case, it follows Rule 1. In the second case the mutation arises at time $\tau_{m}$ after its birth and the new state is either of the form $a^{\prime} b^{\prime} 0$ or 011 . All the concerned states and their evolutions are given in Table 3.

\begin{tabular}{|c|c|c|}
\hline Birth state & Possible evolutions & New state or daughters states \\
\hline & Faithful proliferation $\tau_{2}$ later & \\
\hline & Degradation $\tau_{m}$ later & \\
\hline & Pause $\tau_{m}$ later & \\
\hline & Death $\tau_{m}$ later & \\
\hline & Faithful proliferation $\tau_{2}$ later & \\
\hline & Degradation $\tau_{m}$ later & \\
\hline & Death $\tau_{m}$ later & \\
\hline & Faithful proliferation $\tau_{1}$ later & \\
\hline & Restoration $\tau_{m}$ later & \\
\hline & Degradation $\tau_{m}$ later & \\
\hline & Pause $\tau_{m}$ later & \\
\hline & Death $\tau_{m}$ later & \\
\hline & Faithful proliferation $\tau_{1}$ later & \\
\hline & Death $\tau_{m}$ later & \\
\hline
\end{tabular}

Table 3: Indirect effects: possible evolutions of a $a b 1$ phenotype (unstable states) with $a=1,2$

Rule 4 (Special case of the 011-type cell). As mentioned before, this state represents a long break in the cell cycle. After a long pause $\tau_{b}$, the cell can proliferate or die, and the new state is stable, i.e. of type ab0, see Table 4. 
Rule 5 (Role of the repair capacity). The repair capacity $c=1$ is inherited by proliferation and can be lost by mutation.

All the possibilities are presented in the graphs depicted in Figure 1 and one example of lineage evolutions is given, see Figure 2.

\begin{tabular}{l|ll}
\hline Initial state & Possible evolutions $\tau_{b}$ later & New state \\
\hline \hline \multirow{2}{*}{011} & Back to a proliferative state & 210 \\
& Cell death & 110 \\
\hline
\end{tabular}

Table 4: Indirect effects: possible evolutions of a 011 phenotype (pause state)

\section{The cell population model}

\subsection{Quantitative results}

\begin{tabular}{|c|c|c|}
\hline Symb. & Definition & Domain \\
\hline$n_{0}$ & Initial number of 200-type cells & $\mathbb{N}$ \\
\hline & Initial number of 210-type cells & $\mathbb{N}$ \\
\hline & Discrete time variable & $\mathbb{N}$ \\
\hline$y(k)$ & Mean number of living cells & $\mathbb{R}_{+}$ \\
\hline$y_{d}(k)$ & Mean number of cells damaged by the radiation & $\mathbb{R}_{+}$ \\
\hline$y_{u}(k)$ & Mean number of undamaged cells & $\mathbb{R}_{+}$ \\
\hline \multirow{5}{*}{$\begin{array}{l}\bar{x}_{*}^{110}(k) \\
\bar{x}_{* 00}^{100}(k) \\
\bar{x}_{*}^{011}(k)\end{array}$} & State variables & \\
\hline & Mean number of cells initially in state $110 \&$ in state $* \neq 000$ at time $k$ & $\mathbb{R}_{+}$ \\
\hline & Mean number of cells initially in state $100 \&$ in state $* \neq 000$ at time $k$ & $\mathbb{R}_{+}$ \\
\hline & Mean number of cells initially in state $011 \&$ in state $* \neq 000$ at time $k$ & $\mathbb{R}_{+}$ \\
\hline & Time constants & \\
\hline$\tau_{d}=1$ & Mean response time (hour) of the first damages due to the direct treatment effects & $\mathbb{N}$ \\
\hline$\tau_{1}=36$ & Mean length (hour) of the extended cell cycle & $\mathbb{N}$ \\
\hline$\tau_{2}=24$ & Mean cell cycle length (hour) & $\mathbb{N}$ \\
\hline$\tau_{m}=1$ & Mutation period (hour) & $\mathbb{N}$ \\
\hline$\tau_{b}=75$ & Long break in the cell cycle(hour) & $\mathbb{N}$ \\
\hline \multirow{4}{*}{$p_{\omega}^{\alpha}$} & Probabilities parameters for undamaged cells & \\
\hline & Probability of state transition from $\alpha$ to $\omega$ & {$[0 ; 1]$} \\
\hline & $\alpha$ : initial state & $\{200 ; 210\}$ \\
\hline & $\omega:$ final state & $\{000 ; 011 ; 100 ; 110 ; 2\}$ \\
\hline \multirow{4}{*}{$p_{\omega^{\prime}}^{\alpha^{\prime}}$} & Probabilities parameters for direct \& indirect treatment effects & \\
\hline & Probability of state transition from $\alpha^{\prime}$ to $\omega^{\prime}$ & {$[0 ; 1]$} \\
\hline & $\alpha^{\prime}:$ initial state & $\{011 ; 101 ; 111 ; 201 ; 211\}$ \\
\hline & $\omega^{\prime}$ : final state & $\{000 ; 011 ; 100 ; 101 ; 110 ; 111 ; 201 ; 210 ; 211\}$ \\
\hline
\end{tabular}

Table 5: Table of Notations

We suppose there are $n_{0}$ cells in state 200 and $n_{1}$ cells in state 210 just before the treatment. All the cells behave independently from each other and each cell evolves as a multitype branching process described in the previous section. We calculate the number of living cells at times $k=0, \cdots, n-1$ where $n$ denotes the time range of the experiment. We recall that $k=0$ denotes the end of the treatment session. The average cell population is split up into two parts:

$$
y(k)=y_{u}(k)+y_{d}(k)
$$


where $y_{u}(k)$ and $y_{d}(k)$ are the average number of undamaged and damaged cells respectively.

We begin with the size of the undamaged cell population. Let $p_{2}^{210}\left(\right.$ resp. $\left.p_{2}^{200}\right)$ denote the transition probability from state 210 (resp. 200) to 2 .

\section{Proposition 3.1.}

$$
y_{u}(k)=\left(n_{1} p_{2}^{210}+n_{0} p_{2}^{200}\right)\left(\left(1-k / \tau_{2}\right)_{+}+2^{\kappa \vee 1}\left(\frac{k}{\tau_{2}}-(\kappa-1)_{+}\right)\right)
$$

where $w_{+}$is the positive part of $w\left(w_{+}=w\right.$ if $w \geq 0$ and 0 otherwise), $\kappa \vee 1=\sup (\kappa, 1), \kappa=\left\lfloor k / \tau_{2}\right\rfloor$ is the cell cycle number and $\lfloor x\rfloor$ denotes the integer part of $x$.

We now deal with the size $y_{d}(k)$ of the damaged population. Let us introduce few notations related to our branching process modeling the direct and the indirect effects of the radiotherapy. The real number $p_{\alpha \beta \gamma}^{a b c}$ stands for the transition probability from state $a b c$ to $\alpha \beta \gamma$. The composition of the population of cells is given by the family of stochastic processes $\left(x_{\alpha \beta \gamma}^{a b c}(k)\right)_{k \geq 0}$ where $x_{\alpha \beta \gamma}^{a b c}(k)$ is the number of cells of type $\alpha \beta \gamma$ at time $k$, when the branching process starts with a unique cell in state $a b c$. We mainly focus on the average $\bar{x}_{\alpha \beta \gamma}^{a b c}(k)$ of $x_{\alpha \beta \gamma}^{a b c}(k)$, i.e. $\bar{x}_{\alpha \beta \gamma}^{a b c}(k):=\mathbb{E}\left(x_{\alpha \beta \gamma}^{a b c}(k)\right)$.

Our quantitative analysis allows us to calculate the mean number $\bar{x}_{*}^{a b c}(k)$ of cells still alive at time $k$, when the unique ancestor cell is in state $a b c$. Then, according to the previous notations,

$$
x_{*}^{a b c}(k)=\sum_{\alpha \beta \gamma \neq 000} x_{\alpha \beta \gamma}^{a b c}(k), \quad \bar{x}_{*}^{a b c}(k)=\sum_{\alpha \beta \gamma \neq 000} \bar{x}_{\alpha \beta \gamma}^{a b c}(k) .
$$

We express $y_{d}(k)$ as a linear combination of $\bar{x}_{*}^{110}(k-1), \bar{x}_{*}^{100}(k-1)$ and $\bar{x}_{*}^{011}(k-1)$.

\section{Proposition 3.2.}

$$
y_{d}(k)=n_{1} p_{110}^{210} \bar{x}_{*}^{110}(k-1)+\left(n_{1} p_{100}^{210}+n_{0} p_{100}^{200}\right) \bar{x}_{*}^{100}(k-1)+n_{1} p_{011}^{210} \bar{x}_{*}^{011}(k-1)
$$

Note that (3.3) results from the direct effect of the therapy. Proposition 3.2 obviously implies that we have to calculate $\bar{x}_{*}^{110}(k-1), \bar{x}_{*}^{100}(k-1)$ and $\bar{x}_{*}^{011}(k-1)$ to get $y_{d}(k)$. Their calculations use intensively the mechanism of branching associated with the indirect effects of the therapy. We introduce linear operators acting on sequences $(x(k))_{k \geq 0}$. In particular, $I_{d}$ stands for the identity operator and $q^{-1}$ is the back shift (delay) operator:

$$
q^{-1} x(k)=x(k-1), \quad \forall k \geq 1, \quad q^{-1} x(0):=0 .
$$

The families of all needed operators $\left(\Gamma_{i}\right)_{1 \leq i \leq 7}$ and specific functions $\left(z_{i}\right)_{1 \leq i \leq 15}$ are defined in Section 3.2. 
Proposition 3.3. The quantities $\bar{x}_{*}^{011}(k), \bar{x}_{*}^{100}(k)$ and $\bar{x}_{*}^{110}(k)$ are given by:

$$
\begin{aligned}
& \bar{x}_{*}^{011}(k)=G_{011}(q) z_{12}(k) \\
& \bar{x}_{*}^{100}(k)=\mathbb{1}_{\left[0 ; \tau_{1}[\right.}(k)+G_{100}(q) z_{0}(k) \\
& \bar{x}_{*}^{110}(k)=G_{110}(q) z_{15}(k)
\end{aligned}
$$

where the transfer operators $G_{011}(q), G_{100}(q)$ and $G_{110}(q)$ are defined as follows:

$$
\begin{aligned}
G_{011}(q):= & \left(I_{d}-\Gamma_{5}(q)\right)^{-1}, \quad G_{110}(q):=\left(I_{d}-\Gamma_{7}(q)\right)^{-1} \\
& G_{100}(q):=2 \sum_{i \geq 1}\left(2 p_{101}^{101}\right)^{i-1} q^{-i \tau_{1}} .
\end{aligned}
$$

Our approach generates more results than the ones given in Proposition 3.3, since we are able to calculate all the $\bar{x}_{*}^{a b c}$.

Proposition 3.4. The others $\bar{x}_{*}^{a b c}(k)$ are given by:

$$
\begin{gathered}
\bar{x}_{*}^{211}=\left(I_{d}-\Gamma_{6}(q)\right)^{-1} z_{13}, \quad \bar{x}_{*}^{210}=\mathbb{1}_{\left[0 ; \tau_{2}[\right.}+2 q^{-\tau_{2}}\left(I_{d}-\Gamma_{6}(q)\right)^{-1} z_{13}, \quad \bar{x}_{*}^{201}=z_{7}, \quad \bar{x}_{*}^{200}=z_{8} . \\
\bar{x}_{*}^{111}=\left(I_{d}-\Gamma_{7}(q)\right)^{-1} z_{14}, \quad \bar{x}_{*}^{101}=\sum_{k \geq 0}\left(2 p_{101}^{101}\right)^{k} q^{-k \tau_{1}} z_{0}
\end{gathered}
$$

\subsection{Definition of operators $\Gamma_{\bullet}$ and functions $z_{\bullet}$}

We begin with the family of operators $\left(\Gamma_{i}\right)_{1 \leq i \leq 7}$.

$$
\begin{aligned}
& \Gamma_{1}(q)=2 p_{111}^{111} q^{-\tau_{1}}+2 p_{110}^{011} p_{011}^{111} q^{-\left(\tau_{1}+\tau_{b}+\tau_{m}\right)} \\
& \Gamma_{2}(q)=2 p_{210}^{111} q^{-\left(\tau_{m}+\tau_{2}\right)}+2 p_{210}^{011} p_{011}^{111} q^{-\left(\tau_{2}+\tau_{b}+\tau_{m}\right)} \\
& \Gamma_{3}(q)=2 p_{110}^{011} q^{-\left(\tau_{1}+\tau_{b}\right)} \Gamma_{2}(q)+2 p_{210}^{011} q^{-\left(\tau_{2}+\tau_{b}\right)}\left(I_{d}-\Gamma_{1}(q)\right) \\
& \Gamma_{4}(q)=\Gamma_{1}(q)+2 p_{211}^{211} q^{-\tau_{2}}-2 p_{211}^{211} q^{-\tau_{2}} \Gamma_{1}(q)+2 p_{110}^{211} q^{-\left(\tau_{m}+\tau_{1}\right)} \Gamma_{2}(q) \\
& \Gamma_{5}(q)=\Gamma_{4}(q)+\Gamma_{1}(q)-\Gamma_{1}(q) \Gamma_{4}(q)+p_{011}^{211} \Gamma_{3}(q)\left(1-\Gamma_{1}(q)\right) q^{-\tau_{m}} \\
& \Gamma_{6}(q)=\Gamma_{4}(q)+\Gamma_{5}(q)-\Gamma_{4}(q) \Gamma_{5}(q) \\
& \Gamma_{7}(q)=\Gamma_{6}(q)+\Gamma_{1}(q)-\Gamma_{1}(q) \Gamma_{6}(q)
\end{aligned}
$$

Write $\mathbb{1}_{[a ; b[}(k)$ the rectangular function that is equal to 1 if $k \in[a ; b[$ and 0 otherwise. Let us define the 
functions $z_{\bullet}(k)$.

$$
\begin{aligned}
& z_{0}(k)=\mathbb{1}_{\left[0 ; \tau_{m}[\right.}(k)+p_{101}^{101} \mathbb{1}_{\left[\tau_{m} ; \tau_{1}[\right.}(k) \\
& z_{1}(k)=\mathbb{1}_{\left[0 ; \tau_{b}[\right.}(k)+p_{110}^{011} \mathbb{1}_{\left[\tau_{b} ; \tau_{b}+\tau_{1}[\right.}(k) \\
& z_{2}(k)=z_{1}(k)+p_{210}^{011} \mathbb{1}_{\left[\tau_{b} ; \tau_{b}+\tau_{2}[\right.}(k) \\
& z_{3}(k)=\mathbb{1}_{\left[0 ; \tau_{m}[\right.}(k)+p_{111}^{111} \mathbb{1}_{\left[\tau_{m} ; \tau_{1}[\right.}(k)+p_{210}^{111} \mathbb{1}_{\left[\tau_{m} ; \tau_{m}+\tau_{2}[\right.}(k)+p_{011}^{111} q^{-\tau_{m}} z_{2}+p_{100}^{111} q^{-\tau_{m}} z_{4} \\
& z_{4}(k)=\mathbb{1}_{\left[0 ; \tau_{1}[\right.}(k)+2 \sum_{i \geq 1}\left(2 p_{101}^{101}\right)^{i-1} q^{-i \tau_{1}} z_{0}(k) \\
& z_{5}(k)=2 p_{110}^{011} q^{-\left(\tau_{b}+\tau_{1}\right)} z_{3}(k)+\left(I_{d}-\Gamma_{1}(q)\right) z_{2}(k) \\
& z_{6}(k)=\mathbb{1}_{\left[0 ; \tau_{m}[\right.}(k)+p_{201}^{201} \mathbb{1}_{\left[\tau_{m} ; \tau_{2}[\right.}(k)+p_{100}^{201} q^{-\tau_{m}} z_{4}(k) \\
& z_{7}(k)=\left(I_{d}-2 p_{201}^{201} q^{-\tau_{2}}\right)^{-1} z_{6}(k) \\
& z_{8}(k)=\mathbb{1}_{\left[0 ; \tau_{2}[\right.}(k)+2 q^{-\tau_{2}} z_{7}(k) \\
& z_{9}(k)=\mathbb{1}_{\left[0 ; \tau_{m}[\right.}(k)+p_{200}^{211} q^{-\tau_{m}} z_{8}(k)+p_{100}^{211} q^{-\tau_{m}} z_{4}(k)+p_{211}^{211} \mathbb{1}_{\left[\tau_{m} ; \tau_{2}[\right.}(k) \\
& z_{10}(k)=z_{9}(k)+p_{110}^{211} \mathbb{1}_{\left[\tau_{m} ; \tau_{1}+\tau_{m}[\right.}(k) \\
& z_{11}(k)=2 p_{110}^{211} q^{-\left(\tau_{m}+\tau_{1}\right)} z_{3}(k)+\left(1-\Gamma_{1}(q)\right) z_{10}(k) \\
& z_{12}(k)=\left(1-\Gamma_{4}(q)\right) z_{5}(k)+\Gamma_{3}(q) z_{11}(k) \\
& z_{13}(k)=p_{011}^{211}\left(1-\Gamma_{1}(q)\right) q^{-\tau_{m}} z_{12}(k)+\left(1-\Gamma_{5}(q)\right) z_{11}(k) \\
& z_{14}(k)=\Gamma_{2}(q) z_{13}(k)+\left(1-\Gamma_{6}(q)\right) z_{3}(k) \\
& z_{15}(k)=2 q^{-\tau_{1}} z_{14}(k)+\left(1-\Gamma_{7}(q)\right) \mathbb{1}_{\left[0 ; \tau_{1}[\right.}(k)
\end{aligned}
$$

\subsection{Additional notations and assumptions}

All the parameters are listed in Tables 5 . We suppose that the time parameters are integers and partially ranked as follows:

$$
\tau_{m}<\tau_{2}, \quad \tau_{d}=1<\tau_{2}<\tau_{1}<\tau_{b}
$$

This technical assumption will play an important role, see Section 6.2. The model is finally composed of 31 parameters that are sum up and defined in Table 5 . Some of the transition probabilities defined in Table 5 
are correlated to each other, according to:

$$
\begin{aligned}
& p_{2}^{210}+p_{110}^{210}+p_{100}^{210}+p_{011}^{210}+p_{000}^{210}=1 \\
& p_{2}^{200}+p_{100}^{200}+p_{000}^{200}=1 \\
& p_{211}^{211}+p_{200}^{211}+p_{110}^{211}+p_{100}^{211}+p_{011}^{211}+p_{000}^{211}=1 \\
& p_{201}^{201}+p_{100}^{201}+p_{000}^{201}=1 \\
& p_{111}^{111}+p_{210}^{111}+p_{100}^{111}+p_{011}^{111}+p_{000}^{111}=1 \\
& p_{101}^{101}+p_{000}^{101}=1 \\
& p_{210}^{011}+p_{110}^{011}+p_{000}^{011}=1 .
\end{aligned}
$$

We have finally 20 free transition probabilities. In practice, we replace $p_{000}^{a b c}$ by $1-\sum_{\alpha \beta \gamma \neq 000} p_{\alpha \beta \gamma}^{a b c}$.

\section{Simulation Results}

The model presented in the previous section has been implemented in Matlab ${ }^{\complement}$. The constants $\tau_{d}, \tau_{1}, \tau_{2}, \tau_{m}, \tau_{b}$ are supposed as identical for all the cells but those simplifying assumptions can lead to abrupt variations in the output variables with the presence of steps and peaks. A moving average filter has thus been added to soften the simulated curves. The values of the model parameters are given in Table 6. Initially, we suppose there are $n_{0}=5 \cdot 10^{3}$ cells in state 200 and $n_{1}=5 \cdot 10^{3}$ cells in state 210 just before the treatment. Those quantities are fully compatible with the number of cells generally used in in vitro assays carried out in P96 microplates.

The complete simulation run takes a few seconds on Matlab. Figure 3 shows the ability of the proposed model to reproduce four main types of biological responses of cell cultures: cytotoxic, cytostatic, antiproliferative and delayed growth. Those four mean responses have been obtained with four different sets of parameter values given in Table 6 .

The anti-proliferative profile, described in Figure 3 by a purple plot, is a mean response in which the final growth is lower than a normal growth pattern (black). It is obtained from the delayed growth response by changing 12 model parameters. We firstly increase the probabilities of damage: $p_{111}^{011}$ (cell cycle blocking), $p_{111}^{100}$ (lack of repair capacity) and the probability of mortality: $p_{201}^{000}$. To compensate a part of the previous damages and mortalities, we slightly increase the probabilities of proliferation: $p_{211}^{211}$ and $p_{211}^{200}$ to maintain a growth trend. By comparing the responses of the states: 110, 111, 201 and 211 in Figure 4, we observe a significant reduction of the proliferation rates in the anti-proliferative case compared with the delayed growth context.

From the anti-proliferative pattern, only 5 model parameters: $p_{211}^{\bullet}$ have been changed to get the cytostatic 
profile. For those five probabilities, we have used the same values than the delayed growth case. In comparison with the anti-proliferative situation, it comes to reduce the proliferation ability of the cells and finally leads to an equilibrium state. Figure 4 presents the responses of the nine state variables in the cytostatic case. It confirms that the kinetics of 110, 111, 201 and 211 reach a quasi-constant value.

Finally, the cytotoxic response profile is obtained by increasing all the probabilities of mortality when the damage state of the cells reaches the levels 011, 101, 111, 201 after indirect effects. As a consequence, Figure 4 shows that all the state variables converge to zero, i.e. complete mortality.

As previously emphasized, the number of parameters involved in this model allows to reproduce a large spectrum of response profiles. Another important feature of our model is the possibility to analyze in depth the fluctuations of the population size by comparing and identifying the cell states that cause those transient changes.

\section{Conclusion}

This article deals with the modeling and simulation of cell culture responses after radiotherapy. We particularly address the issues of cell mutation heterogeneity and its effect on the survival dynamics of the treated populations. Ten mutation phenotypes have been considered and the population dynamics is described by an age-dependent multi-type branching process in which each cell is represented by a finite number of mutation states with possible transitions between them. The proposed model relies on five biological rules describing the disturbing effects of radiation on the cell lineage. From this representation, we have formulated the average size of the global survival population as well as the one of the 10 subpopulations. However, it has been intractable to determine the explicit formulas of variances. Our model has been implemented into Matlab ${ }^{\circledR}$ to carry out numerical experiments for different sets of model parameters.

This approach to take the cell heterogeneity into account has several advantages. Firstly, it includes some biological knowledge in terms of proliferation, damage repair capacity and instability. This prior knowledge is represented by five basic rules that are meaningful for biologists. The proposed model is based on two scales: the individual cell level and the population stage. By accounting for the heterogeneity of mutations in each cell, the resulting model becomes more appropriate than lumped parameter models, such as stochastic differential equations, to describe and assess its consequences on the treatment outcome. Moreover, its computation cost remains very low compared with Monte-Carlo simulation techniques requiring several thousands of runs. Another advantage is to estimate the impact and role of each mutation subpopulation. This possibility allows the researcher to test several working assumptions and so explain the impact of some specific intermediate damages on the global survival response. This new model could be applied to other problems in which cell heterogeneity plays a crucial role.

One of the main perspective issue is now to study the parameter identifiability and then to propose a 


\begin{tabular}{|c|c|c|c|c|}
\hline & \multicolumn{4}{|c|}{ Models } \\
\hline Param. & Cytotoxic & Cytostatic & Anti-Proliferative & Delayed Growth \\
\hline$\tau_{d}$ & 1 & 1 & 1 & 1 \\
\hline$\tau_{1}$ & 36 & 36 & 36 & 36 \\
\hline$\tau_{2}$ & 24 & 24 & 24 & 24 \\
\hline$\tau_{m}$ & 1 & 1 & 1 & 1 \\
\hline$\tau_{b}$ & 75 & 75 & 75 & 75 \\
\hline$p_{2}^{200}$ & 2 & 2 & 2 & 2 \\
\hline$p_{100}^{200}$ & 0.6 & 0.6 & 0.6 & 0.6 \\
\hline$p_{000}^{200}$ & 0.4 & 0.4 & 0.4 & 0.4 \\
\hline$p_{2}^{210}$ & 0 & 0 & 0 & 0 \\
\hline$p_{110}^{210}$ & 0.25 & 0.25 & 0.25 & 0.25 \\
\hline$p_{100}^{210}$ & 0.25 & 0.25 & 0.25 & 0.25 \\
\hline$p_{011}^{210}$ & 0.25 & 0.25 & 0.25 & 0.25 \\
\hline$p_{000}^{211}$ & 0.25 & 0.25 & 0.25 & 0.25 \\
\hline$p_{000}^{011}$ & 0.5 & 0.34 & 0.34 & 0.34 \\
\hline$p_{110}^{011}$ & 0.25 & 0.33 & 0.33 & 0.33 \\
\hline$p_{210}^{011}$ & 0.25 & 0.33 & 0.33 & 0.33 \\
\hline$p_{101}^{101}$ & 0.1 & 0.4 & 0.4 & 0.4 \\
\hline$p_{000}^{101}$ & 0.9 & 0.6 & 0.6 & 0.6 \\
\hline$p_{000}^{111}$ & 0.6 & 0.2 & 0.2 & 0.2 \\
\hline$p_{011}^{111}$ & 0.2 & 0.2 & 0.2 & 0.1 \\
\hline$p_{100}^{111}$ & 0.2 & 0.2 & 0.2 & 0.1 \\
\hline$p_{111}^{111}$ & 0 & 0.2 & 0.2 & 0.4 \\
\hline$p_{210}^{111}$ & 0 & 0.2 & 0.2 & 0.2 \\
\hline$p_{000}^{201}$ & 0.7 & 0.4 & 0.4 & 0 \\
\hline$p_{201}^{201}$ & 0 & 0.2 & 0.2 & 0.5 \\
\hline$p_{100}^{201}$ & 0.3 & 0.4 & 0.4 & 0.5 \\
\hline$p_{000}^{211}$ & 0.6 & 0 & 0 & 0 \\
\hline$p_{011}^{211}$ & 0.1 & 0.2 & 0.1 & 0.2 \\
\hline$p_{100}^{211}$ & 0.1 & 0.2 & 0.1 & 0.2 \\
\hline$p_{110}^{211}$ & 0.1 & 0.2 & 0.1 & 0.2 \\
\hline$p_{200}^{211}$ & 0.1 & 0.2 & 0.3 & 0.2 \\
\hline$p_{211}^{211}$ & 0 & 0.2 & 0.4 & 0.2 \\
\hline
\end{tabular}

Table 6: Values of the model parameters for the four types of response.

parameter estimation method from real in vitro data provided by realtime assays such as the cell impedance measurement technology.

\section{Proofs}

\subsection{Proof of Proposition 3.1}

Consider a cell of type either 210 or 200 which is not damaged by the treatment and therefore begins to proliferate at time $T$. This cell gives birth to two new cells at each time $T+m \tau_{2}$, where $m \geq 0$ is an integer. Then, the number $\theta_{0}(k)$ of all the descendants at time $k$ is constant over any interval of the type 
]$T+m \tau_{2}, T+(m+1) \tau_{2}[$ and

$$
\theta_{0}(k)=\mathbb{1}_{\{k<T\}}+22^{\left\lfloor\frac{k-T}{\tau_{2}}\right\rfloor} 1_{\{k \geq T\}}
$$

since:

$$
T+m \tau_{2} \leq k<T+(m+1) \tau_{2} \quad \Leftrightarrow \quad m \leq \frac{k-T}{\tau_{2}}<m+1 \quad \Leftrightarrow \quad m=\left\lfloor\frac{k-T}{\tau_{2}}\right\rfloor .
$$

Recall that $T$ is a random variable with uniform distribution over $\left[0, \tau_{2}\right]$, consequently

$$
E\left(\theta_{0}(k)\right)=\left(1-\frac{k}{\tau_{2}}\right)_{+}+I(k)
$$

where $I(k):=\frac{2}{\tau_{2}} \int_{0}^{\tau_{2} \wedge k} 2^{\left\lfloor\frac{k-u}{\tau_{2}}\right\rfloor} d u$.

Setting $v=(k-u) / \tau_{2}$ we get $I(k)=2 \int_{\left(\frac{k}{\tau_{2}}-1\right)_{+}}^{k / \tau_{2}} 2^{\lfloor v\rfloor} d v$. If $k / \tau_{2}<1$, then $I(k)=2\left(k / \tau_{2}\right)$. Otherwise, setting $\kappa:=\left\lfloor k / \tau_{2}\right\rfloor$ we obtain:

$$
I(k)=2\left(\int_{\frac{k}{\tau_{2}}-1}^{\kappa} 2^{\kappa-1} d v+\int_{\kappa}^{k / \tau_{2}} 2^{\kappa} d v\right)=2^{\kappa}\left(\frac{k}{\tau_{2}}-\kappa+1\right) .
$$

Finally, $I(k)=2^{\kappa \vee 1}\left(\frac{k}{\tau_{2}}-(\kappa-1)_{+}\right)$. Identity (3.2) follows directly.

\subsection{Proof of Proposition 3.2}

It is very convenient to adopt the following convention:

any sequence $(x(k))_{k \geq 0}$ is prolonged to negative index, setting: $x(k)=0, k=-1,-2, \cdots$.

Let us consider a cell of type 210 which either mutates or dies. At time $T$, if it does not dy, according to Table 1, the new state is either 110, or 100 or 011 . Therefore the mean number of cells alive at time $k$ is:

$$
A:=p_{110}^{210} E\left(\bar{x}_{*}^{110}(k-T)\right)+p_{100}^{210} E\left(\bar{x}_{*}^{100}(k-T)\right)++p_{011}^{210} E\left(\bar{x}_{*}^{011}(k-T)\right) .
$$

Recall that $T$ takes its values in $\left[0, \tau_{d}\right]$ with $\tau_{d}=1$, then $k-1<k-T<k$ almost surely. Since $k$ is an integer, property (3.35) implies that $x_{\alpha \beta \gamma}^{a b c}(t)=x_{\alpha \beta \gamma}^{a b c}(k-1)$ for any $t \in[k-1, k[, k \in \mathbb{N}$. Consequently:

$$
x_{*}^{a b c}(t)=x_{*}^{a b c}(k-1), \quad \forall t \in[k-1, k[, k \in \mathbb{N} .
$$

Consequently, $A=p_{110}^{210} \bar{x}_{*}^{110}(k-1)+p_{100}^{210} \bar{x}_{*}^{100}(k-1)+p_{011}^{210} \bar{x}_{*}^{011}(k-1)$.

Similarly, if the cell is initially in state 200 , the mean number of cells alive at time $k$ equals $p_{100}^{200} \bar{x}_{*}^{100}(k-1)$. 


\subsection{Proof of Propositions 3.3 and 3.4}

We will use intensively the following result.

Lemma 6.1. Let $(\varphi(k))_{n \geq 0}$ be a sequence of real numbers, $\lambda \in \mathbb{R}$ and $a \in \mathbb{N}$. Then, the unique solution $\varphi$ of the equation:

$$
\varphi=\varphi_{0}+\lambda q^{-a} \varphi
$$

is $\varphi=\left(I_{d}-\lambda q^{-a}\right)^{-1} \varphi_{0}=\sum_{k \geq 0} \lambda^{k} q^{-k a} \varphi_{0}$.

Note that according to our convention (6.43), the above sum is actually finite. Identity (6.45) follows from standard analysis.

The first term which is easy to calculate is $\bar{x}_{*}^{100}$. By Table 3 and Lemma 6.1 , we get:

$$
\bar{x}_{*}^{101}=p_{101}^{101}\left(\mathbb{1}_{\left[0 ; \tau_{1}[\right.}+2 q^{-\tau_{1}} \bar{x}_{*}^{101}\right)+\left(1-p_{101}^{101}\right) \mathbb{1}_{\left[0 ; \tau_{m}[\right.}=z_{0}+2 p_{101}^{101} q^{-\tau_{1}} \bar{x}_{*}^{101}
$$

and

$$
\bar{x}_{*}^{101}=\sum_{k \geq 0}\left(2 p_{101}^{101}\right)^{k} q^{-k \tau_{1}} z_{0} .
$$

Using Table 2 we have: :

$$
\bar{x}_{*}^{100}=\mathbb{1}_{\left[0 ; \tau_{1}[\right.}+2 q^{-\tau_{1}} \bar{x}_{*}^{101}=\mathbb{1}_{\left[0 ; \tau_{1}[\right.}+2 \sum_{k \geq 0}\left(2 p_{101}^{101}\right)^{k} q^{-(k+1) \tau_{1}} z_{0}=z_{4} .
$$

It remains to calculate $\bar{x}_{*}^{110}$ and $\bar{x}_{*}^{011}$. We begin with $\bar{x}_{*}^{011}$.

Lemma 6.2. We have:

$$
\bar{x}_{*}^{011}=z_{2}+2 p_{210}^{011} q^{-\tau_{2}-\tau_{b}} \bar{x}_{*}^{211}+2 p_{110}^{011} q^{-\tau_{1}-\tau_{b}} \bar{x}_{*}^{111} .
$$

\section{Proof}

According to Table 4:

$$
\begin{aligned}
\bar{x}_{*}^{011} & =p_{210}^{011}\left(\mathbb{1}_{\left[0 ; \tau_{b}[\right.}+q^{-\tau_{b}} \bar{x}_{*}^{210}\right)+p_{110}^{011}\left(\mathbb{1}_{\left[0 ; \tau_{b}[\right.}+q^{-\tau_{b}} \bar{x}_{*}^{110}\right)+\left(1-p_{210}^{011}-p_{110}^{011}\right) \mathbb{1}_{\left[0 ; \tau_{b}[\right.} \\
& =\mathbb{1}_{\left[0 ; \tau_{b}[\right.}+p_{210}^{011} q^{-\tau_{b}} \bar{x}_{*}^{210}+p_{110}^{011} q^{-\tau_{b}} \bar{x}_{*}^{110} .
\end{aligned}
$$


Using Table 2, we get:

$$
\bar{x}_{*}^{110}=\mathbb{1}_{\left[0 ; \tau_{1}[\right.}+2 q^{-\tau_{1}} \bar{x}_{*}^{111} .
$$

Then,

$$
\bar{x}_{*}^{011}=z_{1}+p_{210}^{011} q^{-\tau_{b}} \bar{x}_{*}^{210}+2 p_{110}^{011} q^{-\tau_{1}-\tau_{b}} \bar{x}_{*}^{111} .
$$

From Table 2, we deduce:

$$
\bar{x}_{*}^{210}=\mathbb{1}_{\left[0 ; \tau_{2}[\right.}+2 q^{-\tau_{2}} \bar{x}_{*}^{211} .
$$

Then, (6.47) follows from (6.50) and the above identity. Note that (6.51) implies the second identity in (3.10).

Unfortunately, (6.47) is not a closed form. We express $\bar{x}_{*}^{111}$ in terms of $\bar{x}_{*}^{211}$ in Lemma 6.3 below and we will go back to $\bar{x}_{*}^{011}$ in Lemma 6.4 .

Lemma 6.3. We have:

$$
\left(I_{d}-\Gamma_{1}(q)\right) \bar{x}_{*}^{111}=z_{3}+\Gamma_{2}(q) \bar{x}_{*}^{211} .
$$

\section{Proof}

According to Table 3, we have:

$$
\bar{x}_{*}^{111}=\mathbb{1}_{\left[0 ; \tau_{m}[\right.}+p_{111}^{111}\left(\mathbb{1}_{\left[\tau_{m} ; \tau_{1}[\right.}+2 q^{-\tau_{1}} \bar{x}_{*}^{111}\right)+p_{210}^{111} q^{-\tau_{m}} \bar{x}_{*}^{210}+p_{100}^{111} q^{-\tau_{m}} \bar{x}_{*}^{100}+p_{011}^{111} q^{-\tau_{m}} \bar{x}_{*}^{011} .
$$

We replace $\bar{x}_{*}^{210}$ using $(6.51)$, we get:

$$
\begin{gathered}
\bar{x}_{*}^{111}=\mathbb{1}_{\left[0 ; \tau_{m}[\right.}+p_{111}^{111} \mathbb{1}_{\left[\tau_{m} ; \tau_{1}[\right.}+2 p_{111}^{111} q^{-\tau_{1}} \bar{x}_{*}^{111}+p_{210}^{111} \mathbb{1}_{\left[\tau_{m} ; \tau_{m}+\tau_{2}[\right.}+2 p_{210}^{111} q^{-\tau_{m}-\tau_{2}} \bar{x}_{*}^{211} \\
+p_{100}^{111} q^{-\tau_{m}} \bar{x}_{*}^{100}+p_{011}^{111} q^{-\tau_{m}} \bar{x}_{*}^{011}
\end{gathered}
$$

Using (6.47), we get:

$$
\begin{gathered}
\bar{x}_{*}^{111}=\mathbb{1}_{\left[0 ; \tau_{m}[\right.}+p_{111}^{111} \mathbb{1}_{\left[\tau_{m} ; \tau_{1}[\right.}+2 p_{111}^{111} q^{-\tau_{1}} \bar{x}_{*}^{111}+p_{210}^{111} \mathbb{1}_{\left[\tau_{m} ; \tau_{m}+\tau_{2}[\right.}+2 p_{210}^{111} q^{-\tau_{m}-\tau_{2}} \bar{x}_{*}^{211} \\
+p_{100}^{111} q^{-\tau_{m}} \bar{x}_{*}^{100}+p_{011}^{111} q^{-\tau_{m}}\left(z_{2}+2 p_{210}^{011} q^{-\tau_{2}-\tau_{b}} \bar{x}_{*}^{211}+2 p_{110}^{011} q^{-\tau_{1}-\tau_{b}} \bar{x}_{*}^{111}\right) \\
\bar{x}_{*}^{111}=z_{3}+\left(2 p_{111}^{111} q^{-\tau_{1}}+2 p_{110}^{011} p_{011}^{111} q^{-\tau_{1}-\tau_{b}-\tau_{m}}\right) \bar{x}_{*}^{111}+2\left(p_{210}^{111} q^{-\tau_{m}-\tau_{2}}+p_{011}^{111} p_{210}^{011} q^{-\tau_{2}-\tau_{b}-\tau_{m}}\right) \bar{x}_{*}^{211} .
\end{gathered}
$$


Lemma 6.4. We have:

$$
\left(I_{d}-\Gamma_{1}(q)\right) \bar{x}_{*}^{011}=z_{5}+\Gamma_{3}(q) \bar{x}_{*}^{211} .
$$

\section{Proof}

We eliminate $\bar{x}_{*}^{111}$ with (6.52) and (6.47), namely we apply : $2 p_{110}^{011} q^{-\tau_{1}-\tau_{b}} \times(6.52)+\left(I_{d}-\Gamma_{1}(q)\right) \times(6.47)$. After simplifications, we get:

$$
\left(I_{d}-\Gamma_{1}(q)\right) \bar{x}_{*}^{011}=2 p_{110}^{011} q^{-\tau_{1}-\tau_{b}}\left(z_{3}+\Gamma_{2}(q) \bar{x}_{*}^{211}\right)+\left(I_{d}-\Gamma_{1}(q)\right)\left(z_{2}+2 p_{210}^{011} q^{-\tau_{2}-\tau_{b}} \bar{x}_{*}^{211}\right) .
$$

Lemma 6.5. We have:

$$
\left(I_{d}-\Gamma_{4}(q)\right) \bar{x}_{*}^{211}=z_{11}+p_{011}^{211} q^{-\tau_{m}}\left(I_{d}-\Gamma_{1}(q)\right) \bar{x}_{*}^{011} .
$$

\section{Proof}

Using the branching properties induced by Table 3, we get:

$$
\begin{gathered}
\bar{x}_{*}^{211}=\mathbb{1}_{\left[0 ; \tau_{m}[\right.}+p_{200}^{211} q^{-\tau_{m}} \bar{x}_{*}^{200}+p_{110}^{211} q^{-\tau_{m}} \bar{x}_{*}^{110}+p_{100}^{211} q^{-\tau_{m}} \bar{x}_{*}^{100}+p_{011}^{211} q^{-\tau_{m}} \bar{x}_{*}^{011} \\
+p_{211}^{211}\left(\mathbb{1}_{\left[\tau_{m} ; \tau_{2}[\right.}+2 q^{-\tau_{2}} \bar{x}_{*}^{211}\right) \\
\bar{x}_{*}^{201}=\mathbb{1}_{\left[0 ; \tau_{m}[\right.}+p_{100}^{201} q^{-\tau_{m}} \bar{x}_{*}^{100}+p_{201}^{201}\left(\mathbb{1}_{\left[\tau_{m} ; \tau_{2}[\right.}+2 q^{-\tau_{2}} \bar{x}_{*}^{201}\right) .
\end{gathered}
$$

By Table 2, we have:

$$
\bar{x}_{*}^{200}=\mathbb{1}_{\left[0 ; \tau_{2}[\right.}+2 q^{-\tau_{2}} \bar{x}_{*}^{201}
$$

It is clear that relation (6.56) is equivalent to:

$$
\left(I_{d}-2 p_{201}^{201} q^{-\tau_{2}}\right) \bar{x}_{*}^{201}=z_{6}
$$

or

$$
\bar{x}_{*}^{201}=z_{7} .
$$

Using (6.57), we get:

$$
\bar{x}_{*}^{200}=\mathbb{1}_{\left[0 ; \tau_{2}[\right.}+2 q^{-\tau_{2}} z_{7}=z_{8} .
$$


Note that (6.58) and (6.59) give the two last identities in (3.10). We modify (6.55) using (6.59):

$$
\begin{aligned}
\bar{x}_{*}^{211}= & \mathbb{1}_{\left[0 ; \tau_{m}[\right.}+p_{200}^{211} q^{-\tau_{m}} z_{8}+p_{110}^{211} q^{-\tau_{m}} \bar{x}_{*}^{110}+p_{100}^{211} q^{-\tau_{m}} z_{4} \\
& +p_{011}^{211} q^{-\tau_{m}} \bar{x}_{*}^{011}+p_{211}^{211} \mathbb{1}_{\tau_{m} ; \tau_{2}[}+2 p_{211}^{211} q^{-\tau_{2}} \bar{x}_{*}^{211} \\
= & z_{9}+p_{110}^{211} q^{-\tau_{m}} \bar{x}_{*}^{110}+p_{011}^{211} q^{-\tau_{m}} \bar{x}_{*}^{011}+2 p_{211}^{211} q^{-\tau_{2}} \bar{x}_{*}^{211} .
\end{aligned}
$$

By (6.49) we get:

$$
\bar{x}_{*}^{211}=z_{9}+p_{110}^{211} \mathbb{1}_{\left[\tau_{m} ; \tau_{1}+\tau_{m}[\right.}+2 p_{110}^{211} q^{-\tau_{m}-\tau_{1}} \bar{x}_{*}^{111}+p_{011}^{211} q^{-\tau_{m}} \bar{x}_{*}^{011}+2 p_{211}^{211} q^{-\tau_{2}} \bar{x}_{*}^{211} .
$$

Relation which is equivalent to:

$$
\left(I_{d}-2 p_{211}^{211} q^{-\tau_{2}}\right) \bar{x}_{*}^{211}=z_{10}+2 p_{110}^{211} q^{-\tau_{m}-\tau_{1}} \bar{x}_{*}^{111}+p_{011}^{211} q^{-\tau_{m}} \bar{x}_{*}^{011} .
$$

We eliminate $\bar{x}_{*}^{111}$ doing: $\left(I_{d}-\Gamma_{1}(q)\right) \times(6.60)+2 p_{110}^{211} q^{-\tau_{m}-\tau_{1}} \times(6.52)$ and we simplify:

$$
\begin{aligned}
\left(I_{d}-\Gamma_{1}(q)\right)\left(I_{d}-2 p_{211}^{211} q^{-\tau_{2}}\right) \bar{x}_{*}^{211}= & \left(\left(I_{d}-\Gamma_{1}(q)\right) z_{10}+p_{011}^{211} q^{-\tau_{m}}\left(I_{d}-\Gamma_{1}(q)\right) \bar{x}_{*}^{011}\right. \\
& +2 p_{110}^{211} q^{-\tau_{m}-\tau_{1}} z_{3}+2 p_{110}^{211} q^{-\tau_{m}-\tau_{1}} \Gamma_{2}(q) \bar{x}_{*}^{211} .
\end{aligned}
$$

We are now able to prove (3.5) and (3.7).

Lemma 6.6. We have:

$$
\left(I_{d}-\Gamma_{5}(q)\right) \bar{x}_{*}^{011}=z_{12}, \quad\left(I_{d}-\Gamma_{7}(q)\right) \bar{x}_{*}^{110}=z_{15} .
$$

\section{Proof}

We sum : $\left(I_{d}-\Gamma_{4}(q)\right) \times(6.53)+\Gamma_{3}(q) \times(6.54)$ and we simplify, we get:

$$
\left(I_{d}-\Gamma_{4}(q)\right)\left(I_{d}-\Gamma_{1}(q)\right) \bar{x}_{*}^{011}=\left(I_{d}-\Gamma_{4}(q)\right) z_{5}+\Gamma_{3}(q) z_{11}+p_{011}^{211} q^{-\tau_{m}} \Gamma_{3}(q)\left(I_{d}-\Gamma_{1}(q)\right) \bar{x}_{*}^{011} .
$$

We easily deduce the first relation in (6.61). As for the second identity, the formal operation : $\left(I_{d}-\Gamma_{5}(q)\right) \times$ $(6.54)+p_{011}^{211} q^{-\tau_{m}}\left(I_{d}-\Gamma_{1}(q)\right) \times(6.61)$ permits to eliminate $\bar{x}_{*}^{011}$

$$
\left(I_{d}-\Gamma_{5}(q)\right)\left(I_{d}-\Gamma_{4}(q)\right) \bar{x}_{*}^{211}=\left(I_{d}-\Gamma_{5}(q)\right) z_{11}+p_{011}^{211} q^{-\tau_{m}}\left(I_{d}-\Gamma_{1}(q)\right) z_{12} .
$$


This relation is equivalent to:

$$
\left(I_{d}-\Gamma_{6}(q)\right) \bar{x}_{*}^{211}=z_{13} .
$$

Using " $\left(I_{d}-\Gamma_{6}(q)\right) \times(6.52)+\Gamma_{2}(q) \times(6.62)$ " we obtain:

$$
\left(I_{d}-\Gamma_{6}(q)\right)\left(I_{d}-\Gamma_{1}(q)\right) \bar{x}_{*}^{111}=\left(I_{d}-\Gamma_{6}(q)\right) z_{3}+\Gamma_{2}(q) z_{13} .
$$

or equivalently:

$$
\left(I_{d}-\Gamma_{7}(q)\right) \bar{x}_{*}^{111}=z_{14} .
$$

Simplifying $\left(I_{d}-\Gamma_{7}(q)\right) \times(6.49)+2 q^{-\tau_{1}} \times(6.63)$ leads to:

$$
\left(I_{d}-\Gamma_{7}(q)\right) \bar{x}_{*}^{110}=\left(I_{d}-\Gamma_{7}(q)\right) \mathbb{1}_{\left[0 ; \tau_{1}[\right.}+2 q^{-\tau_{1}} z_{14}=z_{15} .
$$

It is clear that (6.62) (resp. (6.63)) implies the first identity in (3.10) (resp. (3.11)).

\section{References}

[1] G. Albano, V. Giorno, P. Román-Román, and F. Torres-Ruiz. Inferring the effect of therapy on tumors showing stochastic gompertzian growth. Journal of Theoretical Biology, 276(1):67-77, 2011. 4

[2] A.R.A. Anderson and V. Quaranta. Integrative mathematical oncology. Nature Reviews Cancer, $8(3): 227-234,2008.2$

[3] C. A. Athale and T. S. Deisboeck. The effects of egf-receptor density on multiscale tumor growth patterns. J. Theor. Biol., 238:771-779, 2006. 3

[4] C. A. Athale, Y. Mansury, and T. S. Deisboeck. Simulating the impact of a molecular 'decision-process' on cellular phenotype and multicellular patterns in brain tumors. J. Theor. Biol., 233:469-481, 2005. 3

[5] Krishna B. Athreya and Peter E. Ney. Branching processes. Springer-Verlag, New York, 1972. Die Grundlehren der mathematischen Wissenschaften, Band 196. 4

[6] Martina Baar, Loren Coquille, Hannah Mayer, Michael Hoelzel, Meri Rogava, Thomas Tueting, and Anton Bovier. A stochastic model for immunotherapy of cancer. SCIENTIFIC REPORTS, 6, APR 11 2016. 3

[7] HT Banks and S. Hu. Nonlinear stochastic markov processes and modeling uncertainty in populations. Technical report, DTIC Document, 2011. 4 
[8] D. Bresch, T. Colin, E. Grenier, B. Ribba, and O. Saut. A viscoelastic model for avascular tumor growth. Discrete and Continuous Dynamical Systems, Supplement(Issue: Special):101-108, 2009. 3

[9] F. J. Burns and I. F. Tannock. On the existence of a G0 phase in the cell cycle. Cell Tissue Kinet., $3: 321,1970.3$

[10] R. Chen and O. Hyrien. Quasi-and pseudo-maximum likelihood estimators for discretely observed continuous-time markov branching processes. Journal of statistical planning and inference, 141(7):22092227, 2011. 4

[11] O. Chis and D. Opris. Mathematical analysis of stochastic models for tumor-immune systems. $\underline{\operatorname{arXiv}}$ preprint arXiv:0906.2794, 2009. 4

[12] E. B. Cox, M. A. Woodburry, and L. E. Meyers. A new model for tumor growth analysis based on a postulated inhibitory substance. Comp. Biomed. Res., 13:437, 1980. 3

[13] K. Danesh, R. Durrett, L. Havrilesky, and E. Myers. A branching process model of ovarian cancer. Journal of Theoretical Biology, 2012. 4

[14] A. Dawson and T. Hillen. Derivation of the tumour control probability (tcp) from a cell cycle model. Computational and Mathematical Methods in Medicine, 7(2-3):121-141, 2006. 3

[15] D. Drasdo and St. Höhme. A single-cell-based model of tumor growth in vitro: monolayers and spheroids. Phys. Biol., 2:133-147, 2005. 3

[16] R. Durrett, J. Foo, K. Leder, J. Mayberry, and F. Michor. Intratumor heterogeneity in evolutionary models of tumor progression. Genetics, 188(2):461-477, 2011. 4

[17] J. A. Engelberg, Glen E. P. Ropella, and C. A. Hunt. Essential operating principles for tumor spheroid growth. BMC Systems Biology, 2(110):1-19, 2008. 3

[18] J. J. Fischer. Mathematical simulation of radiation theory of solid tumors. I. Calculations. Acta Radiol. Ther. Phys. Biol., 10:73, 1971. 3

[19] J. Galle, G. Aust, G. Schaller, T. Beyer, and D. Drasdo. Individual cell-based models of the spatiotemporal organisation of multicellular systems - achievements and limitations. Cytometry, Cytometry A, 69A:704-710, 2006. 3

[20] G. F. Gause. The struggle for existence. Williams and Wilkins, New York, 1934. 3

[21] H. A. Gay and A. Niemierko. A free program for calculating eud-based ntcp and tcp in external beam radiotherapy. Physica Medica, 23(115-125), 2007. 3 
[22] P. B. Gupta, C. M. Fillmore, G. Jiang, S. D. Shapira, K. Tao, C. Kuperwasser, and E. S. Lander. Stochastic state transitions give rise to phenotypic equilibrium in populations of cancer cells. Cell, 146:633-644, August 19 2011. 2

[23] P.B. Gupta, C.M. Fillmore, G. Jiang, S.D. Shapira, K. Tao, C. Kuperwasser, and E.S. Lander. Stochastic state transitions give rise to phenotypic equilibrium in populations of cancer cells. Cell, 146(4):633-644, 2011. 4

[24] Theodore E. Harris. The theory of branching processes. Die Grundlehren der Mathematischen Wissenschaften, Bd. 119. Springer-Verlag, Berlin, 1963. 4

[25] O. Hyrien, J. Dietrich, and M. Noble. Mathematical and experimental approaches to identify and predict the effects of chemotherapy on neuroglial precursors. Cancer research, 70(24):10051-10059, 2010. 4

[26] J. Istas. Introduction aux modélisations mathématiques pour les sciences du vivant. Springer-Verlag, 2000. 3

[27] Peter Jagers. Branching processes with biological applications. Wiley-Interscience [John Wiley \& Sons], London, 1975. Wiley Series in Probability and Mathematical Statistics-Applied Probability and Statistics. 4

[28] A.A. Katouli and N.L. Komarova. The worst drug rule revisited: mathematical modeling of cyclic cancer treatments. Bulletin of mathematical biology, 73(3):549-584, 2011. 4

[29] R. Keinj, T. Bastogne, and P. Vallois. Tumor growth modeling based on cell and tumor lifespans. Journal of Theoretical Biology, 2012. 4

[30] M. Kimmel and D.E. Axelrod. Branching processes in biology, volume 19. Springer, 2002. 4

[31] C.A.M. La Porta, S. Zapperi, and J.P. Sethna. Senescent cells in growing tumors: population dynamics and cancer stem cells. PLoS computational biology, 8(1):e1002316, 2012. 4

[32] A. K. Laird. Dynamics of tumor growth. Br J of Cancer, 18:490-502, 1964. 3

[33] Jeffrey A Magee, Elena Piskounova, and Sean J Morrison. Cancer stem cells: Impact, heterogeneity, and uncertainty. Cancer Cell, 21(3):283-296, 2012. 2

[34] S. Michelson and J. T. Leith. A survey of Models for Tumor-Immune System Dynamics, chapter Tumor Heterogeneity and Growth Control, pages 295-333. Birkhäuser, 1997. 2

[35] N.E. Navin and J. Hicks. Tracing the tumor lineage. Molecular oncology, 4(3):267-283, 2010. 4 
[36] P.K. Newton, J. Mason, K. Bethel, L.A. Bazhenova, J. Nieva, and P. Kuhn. A stochastic markov chain model to describe lung cancer growth and metastasis. PloS one, 7(4):e34637, 2012. 4

[37] R.E. Nordon, K.H. Ko, R. Odell, and T. Schroeder. Multi-type branching models to describe cell differentiation programs. Journal of Theoretical Biology, 277(1):7-18, 2011. 4

[38] N. Rashevsky. Outline of a mathematical approach to the cancer problem. Bull. Math. Biophys., 7(69), 1945. 3

[39] N. Rashevsky. Mathematical Biophysics. University of Chicago Press, Chicago, 1948. 3

[40] R. K. Sachs, L. R. Hlatky, and P. Hahnfeldt. Simple ODE models of tumor growth and anti-angiogenic or radiation treatment. Mathematical and Computer Modelling, 33:1297-1305, 2001. 3

[41] M. Sehl, H. Zhou, J.S. Sinsheimer, and K.L. Lange. Extinction models for cancer stem cell therapy. Mathematical Biosciences, 2011. 4

[42] M. Shackleton, E. Quintana, E. R. Fearon, and S. J. Morrison. Heterogeneity in cancer: Cancer stem cells versus clonal evolution. Cell, 138:822-839, September 2009. 2

[43] G. G. Steel. Growth Kinetics of Tumors. Oxford: Clarendon Press, 1977. 3

[44] Xiaoqiang Sun, Jiguang Bao, and Yongzhao Shao. Mathematical Modeling of Therapyinduced Cancer Drug Resistance: Connecting Cancer Mechanisms to Population Survival Rates. SCIENTIFIC REPORTS, 6, MAR 1 2016. 3

[45] G. W. Swan. Cancer chemotherapy: optimal control using the Verhulst-pearl equation. Bull. Math. Biol., 48:381, 1986. 3

[46] J. R. Thompson and B. W Brown. Cancer Modeling. Marcel Dekker, 1987. 3

[47] L. von Bertalanffy. Fundamental Aspects of Normal and Malignant Growth, chapter Principles and theory of growth, page 137. Elsevier, Amsterdam, Nowinsky, W. W. edition, 1960. 3

[48] G. Weiss. Multiagent Systems, A Modern Approach to Distributed Artificial Intelligence. MIT Press, 1999. 3

[49] M. Wooldridge. An Introduction to MultiAgent Systems. Wiley and Sons, 2002. 3

[50] M Zaider and G N Minerbo. Tumour control probability: a formulation applicable to any temporal protocol of dose delivery. Physics in Medicine and Biology, 45(2):279-293, 2000. 3 
[51] L. Zhang, C. A. Athale, and T. S. Deisboeck. Development of a three-dimensional multiscale agent-based tumor model: simulating gene-protein interaction profiles, cell phenotypes and multicellular patterns in brain cancer. J. Theor. Biol., 244:96-107, 2007. 3

[52] L. Zhang, C. G. Strouthos, Z. Wang, and T. S. Deisboeck. Simulating brain tumor heterogeneity with a multiscale agent-based model: Linking molecular signatures, phenotypes and expansion rate. Math Comput Model, 49(1-2):307-319, 2009. 3 

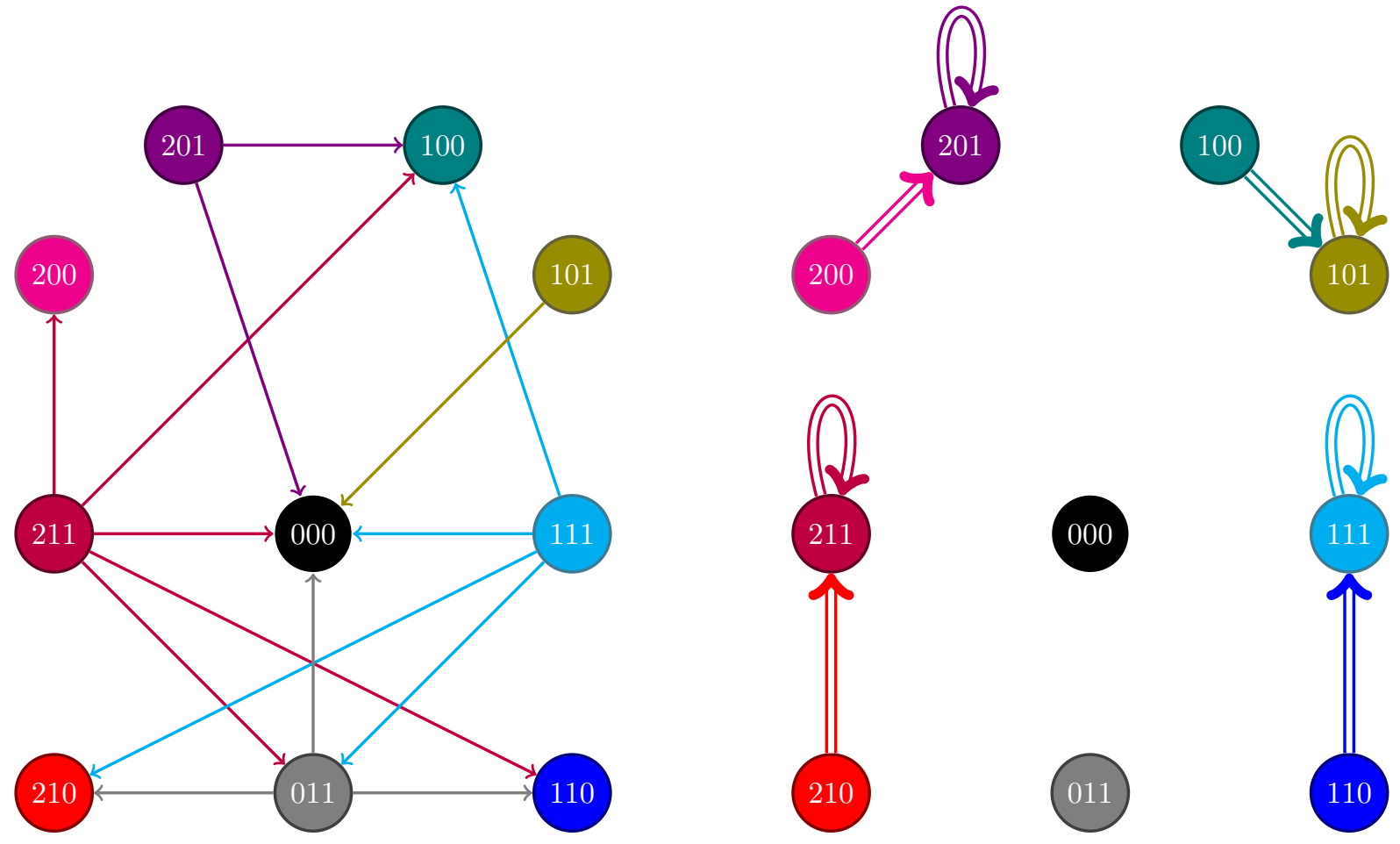

Figure 1: Graphs giving all possibilities of evolutions of damaged cells (indirect effects). On the left-hand side, the graphs expresses the possible state changes. On the right-hand side, an arrow means: "this state may give birth to two cells of type".

\section{Figures}




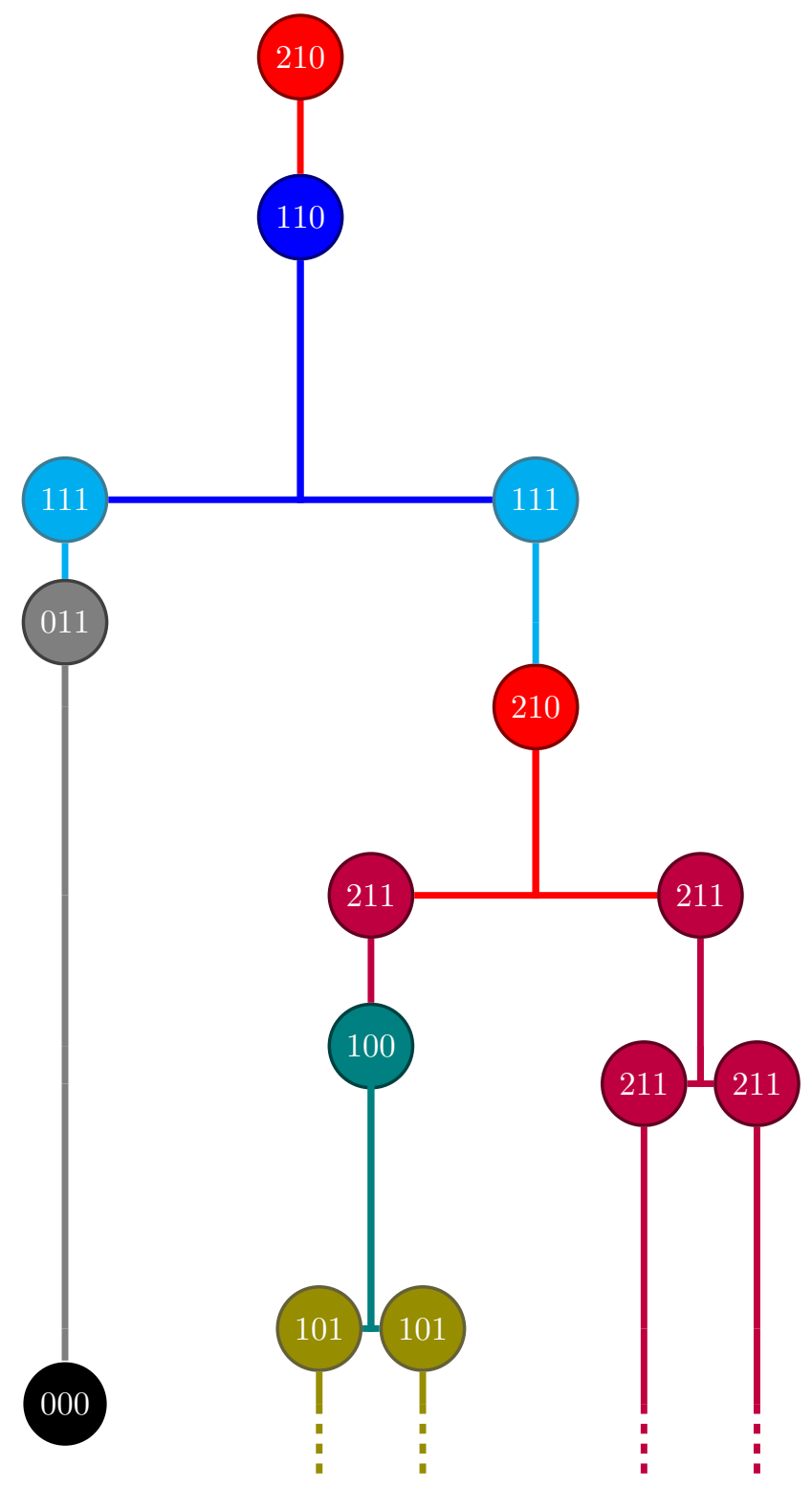

Figure 2: In this example, a 210 type cell is attained by the therapy. It becomes a less aggressive cell 110 that proliferates to give two 111 type cells. One of those dies, while the second one restores. From its new states it gives birth to two 211 type cells. The first one changes to a 100 state that gives birth to two daughters of type 101 . The second one proliferates faithfully... 


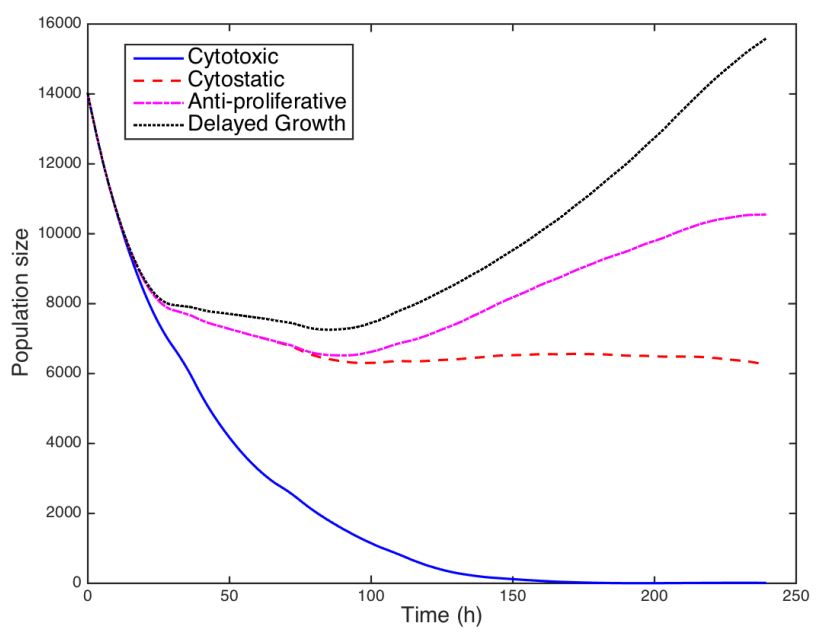

Figure 3: Simulation results of living cell populations. Four main types of biological responses are reproduced, corresponding each to four different sets of parameter values
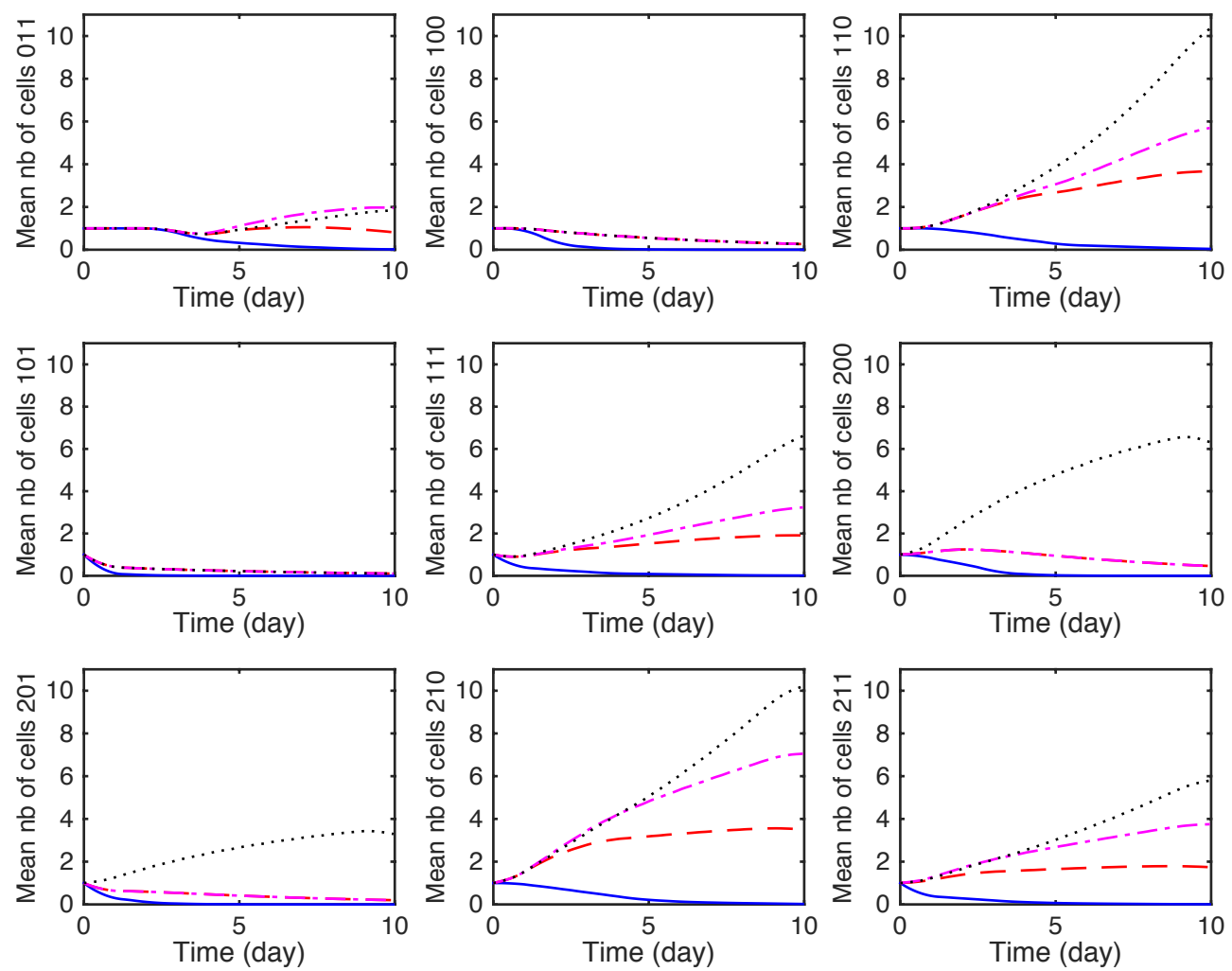

Figure 4: Mutation states for Delayed Growth (black), Anti-proliferative Responses (magenta), Cytostatic (red) and Cytotoxic Responses (blue). Each branching process starts with one cell at time 0. 